\title{
BOUNDS ON COEFFICIENTS OF RECIPROCALS OF FORMAL POWER SERIES WITH RAPIDLY DECREASING COEFFICIENTS
}

\author{
KENNETH S. BERENHAUT, EDWARD E. ALLEN, AND SAM J. FRASER
}

Received 8 August 2006; Revised 19 September 2006; Accepted 26 September 2006

This paper studies reciprocals of formal power series whose coefficients are monotone and bounded by a geometrically decaying sequence. Explicit and applicable, optimal decay rates are provided for the coefficients of the reciprocal series in terms of the parameters of the geometric bound. The results imply a best possible lower bound on the zeros of the series being considered.

Copyright (c) 2006 Kenneth S. Berenhaut et al. This is an open access article distributed under the Creative Commons Attribution License, which permits unrestricted use, distribution, and reproduction in any medium, provided the original work is properly cited.

\section{Introduction}

This paper studies reciprocals of power series whose coefficients are monotone and bounded by a geometrically decaying sequence. In particular, for fixed $A \geq 1$ and $0<$ $r<1$, let the sets $\mathscr{F}_{A, r}$ and $\mathscr{F}_{A, r}^{I}$ be defined by

$$
\begin{gathered}
\mathscr{F}_{A, r} \stackrel{\text { def }}{=}\left\{Q: Q(z)=1+\sum_{k=1}^{\infty} q_{k} z^{k},\left\{q_{k}\right\} \text { is nonincreasing and } 0 \leq q_{i} \leq A r^{i}, \text { for each } i \geq 1\right\}, \\
\mathscr{F}_{A, r}^{I} \stackrel{\text { def }}{=}\left\{\Omega: \Omega(z)=1+\sum_{k=1}^{\infty} \omega_{k} z^{k}, \Omega=\frac{1}{Q}, \text { for some } Q \in \mathscr{F}_{A, r}\right\} .
\end{gathered}
$$

Disregarding its probabilistic context, the well-known Kendall renewal theorem (cf. [2, $16,17,21,27])$ can essentially be restated as follows.

Theorem 1.1 [17]. Suppose $\Omega(z)=\sum_{i=0}^{\infty} \omega_{i} z^{i} \in \mathscr{F}_{A, r}^{I}$, for some $A>0$ and $r<1$. Then,

$$
\left|\omega_{i}\right|=O\left(\sigma^{i}\right)
$$

for some $\sigma<1$.

Here, we take steps towards obtaining an explicit form of Theorem 1.1. Specifically, we will prove the following. 
THEOREM 1.2. Suppose $\Omega \in \mathscr{F}_{A, r}^{I}$, where $A$ and $r$ are constants satisfying

(a) $1 \leq A \leq 1 /(2 r)$,

(b) $\left(A^{2} r^{2}-2 A^{2} r+A\right)(A+(1 / 2) /(A+1 / 2))+A r^{2}-2 A r-A^{2} r^{3}+A^{2} r^{2} \geq 0$,

(c) $\left(-2 A^{2} r+A\right)(A+(1-A r) /(A+1-A r))-A r+A^{2} r^{2} \geq 0$,

(d) $\left((1-A r)\left(A^{2}-A+1-A r\right) /(A+1-A r)\right)+A\left(A^{2}-A+1-A r\right)+A-1-A^{2} r+$ Ar $-A^{2} \geq 0$.

If $\Omega(z)=\sum_{i=0}^{\infty} \omega_{i} z^{i}$, then for $n \geq 1$,

$$
\left|\omega_{n}\right| \leq \frac{s_{1}^{n+1}+(-1)^{n} s_{2}^{n+1}}{\delta} r^{n}=\frac{s_{1}+s_{2}(-1)^{n}\left(s_{2} / s_{1}\right)^{n}}{\delta}\left(s_{1} r\right)^{n} \leq C_{A, r} s_{A, r}^{n}
$$

where $\delta \stackrel{\text { def }}{=} \sqrt{A^{2}+4(1-A r)}, s_{1} \stackrel{\text { def }}{=}(\delta+A) / 2, s_{2} \stackrel{\text { def }}{=}(\delta-A) / 2$,

$$
\begin{aligned}
& s_{A, r} \stackrel{\text { def }}{=} r s_{1}<1, \\
& C_{A, r} \stackrel{\text { def }}{=} \frac{A}{s_{1}} \leq 1 .
\end{aligned}
$$

Note that if $(A, r)$ satisfies $A r<1$, then $s_{A, r}<1$ and (as suggested by Theorem 1.1) the coefficients of the reciprocal series decay at an exponential rate. To see this bound, note that for $0<r<1$ and $0<A r<1$,

$$
1-r s_{1}=\frac{(2-r A)-r \delta}{2}=\frac{(2-r A)^{2}-r^{2} \delta^{2}}{2(2-r A)+2 r \delta}=\frac{2(1-r A)\left(1-r^{2}\right)}{(2-r A)+r \delta}>0 .
$$

It is crucial that for given $A$ and $r$, assumptions (a)-(d) are easily verifiable and are satisfied for a significant portion of the strip $\{(A, r): 0 \leq r \leq 1$ and $A \geq 1\}$. Some pairs $(A, r)$ satisfying the assumptions of Theorem 1.2 are given (in the shaded region) in Figure 1.1.

Monotone sequences and generating functions appear in all facets of applied and pure mathematics, most notably enumerative combinatorics and applied probability (cf. Wilf [34] and Feller [14]). Applications of Theorem 1.2 to quantitative convergence rates for Markov chains will be discussed elsewhere.

For pairs $(A, r)$ satisfying assumptions (a)-(d), Theorem 1.2 gives the optimal value of $\sigma$ in (1.2) which applies for all $\Omega \in \mathscr{F}_{A, r}^{I}$. Consideration of the remaining pairs remains open.

The next example gives zero-free regions for complex power series with rapidly decaying coefficients.

Example 1.3. Theorem 1.2 has immediate implications on lower bounds for the modulus of the smallest zero of a power series $Q \in \mathscr{F}_{A, r}$. Since the result places a lower bound on the radius of convergence $R$ of $\Omega=1 / Q$, via

$$
R=\frac{1}{\limsup _{n \rightarrow \infty} \sqrt[n]{\omega_{n}}} \geq \frac{1}{s_{A, r}}
$$

it also places a lower bound on zeros of Q. Indeed, we have the following corollary. 
Kenneth S. Berenhaut et al. 3

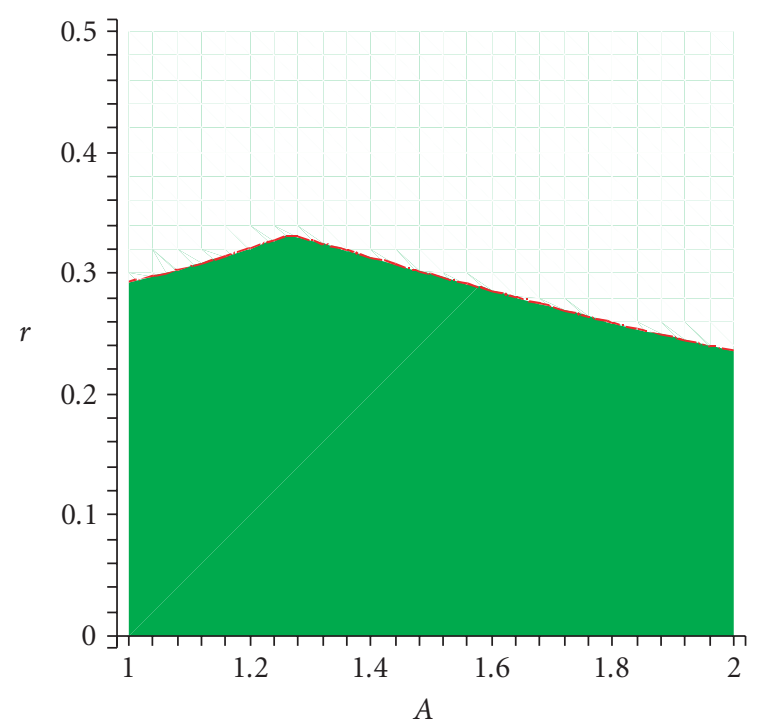

Figure 1.1. Some pairs $(A, r)$ satisfying the assumptions of Theorem 1.2.

Corollary 1.4. Suppose $z_{0}$ is a root of a power series $Q \in \mathscr{F}_{A, r}$, where $Q(z)=1+q_{1} z+$ $q_{2} z^{2}+\cdots$, and $A$ and $r$ satisfy the hypotheses of Theorem 1.2, then

$$
\left|z_{0}\right| \geq s_{A, r}^{-1}
$$

In fact, the series $Q \in \mathscr{F}_{A, r}$ given by

$$
\begin{aligned}
Q(z) & =1+A r z+A r^{3} z^{2}+A r^{3} z^{3}+A r^{5} z^{4}+A r^{5} z^{5}+A r^{7} z^{6}+\cdots \\
& =1+\frac{A r z\left(1+r^{2} z\right)}{1-r^{2} z^{2}}=\frac{1-r^{2} z^{2}+A r z\left(1+r^{2} z\right)}{1-r^{2} z^{2}} \\
& =\frac{(A r-1)(r z)^{2}+A(r z)+1}{1-r^{2} z^{2}}
\end{aligned}
$$

has a zero at $z=-s_{A, r}^{-1}$, and Corollary 1.4 is optimal. The series in (1.8) also serves to show that the decay rate $s_{A, r}$ of Theorem 1.2 is optimal in that context as well.

Power series with restricted coefficients have been studied in the context of determining distributions of zeros (cf. Flatto et al. [15], Solomyak [26], Beaucoup et al. [3, 4], and Pinner [24]). Related problems for polynomials have been considered by Odlyzko and Poonen [23], Yamamoto [36], Borwein and Pinner [12], Borwein and Erdélyi [11], and others.

Berenhaut and Morton [10] provide a result along the lines of Theorem 1.2, when the monotonicity assumption is dropped by studying the recurrence in (2.11), below. Figure 1.2 compares the decay rate $s_{1, r}$ (i.e., with $A=1$ ) of Theorem 1.2 with the comparable rate bound from [10] (for $r$ near $1 / 4$ ). 
4 Reciprocals of formal power series

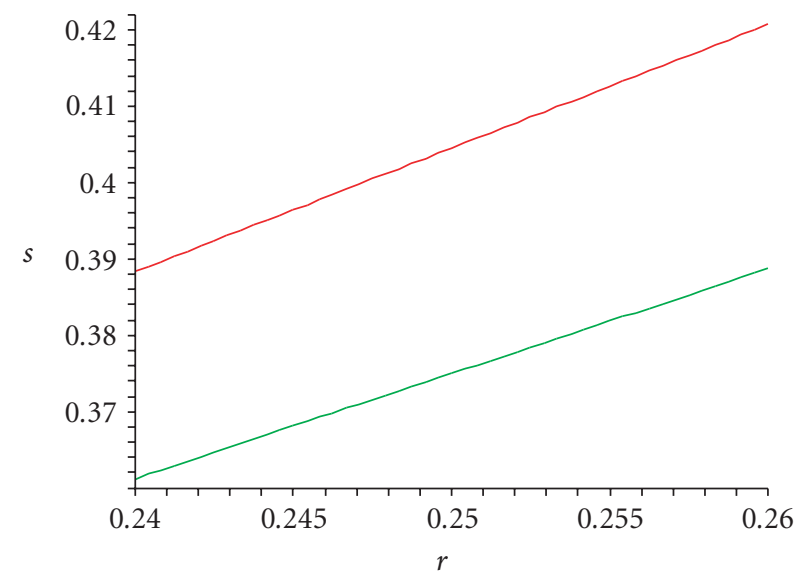

Figure 1.2. A comparison of the decay rates $s_{1, r}$ of Theorem 1.2 with the comparable rates from [10]. The lower and upper curves give the optimal rates with and without the assumption of monotonicity of coefficients, respectively.

The remainder of the paper proceeds as follows. Section 2 contains some preliminary notation and the statement of a crucial lemma (Lemma 2.1) on linear recurrences. Section 3 comprises a discretization approach which serves to limit the scope of possibilities which need to be considered. The paper concludes with a proof of Lemma 2.1 (Section 4). Section 5 contains a proof of a technical lemma employed in Section 3.

\section{Preliminaries}

Suppose $\Omega \in \mathscr{F}_{A, r}^{I}$ and $Q \in \mathscr{F}_{A, r}$ satisfy $\Omega=1 / Q$. In analyzing the coefficients of $\Omega$, it will be convenient to instead consider the coefficients $h_{i}=\omega_{i} / r^{i}$ and $\phi_{i}=q_{i} / r^{i}$ of the series

$$
\begin{aligned}
& \Omega^{*}(z) \stackrel{\text { def }}{=} \Omega\left(\frac{z}{r}\right), \\
& Q^{*}(z) \stackrel{\text { def }}{=} Q\left(\frac{z}{r}\right),
\end{aligned}
$$

respectively.

Note that $0 \leq \phi_{i} \leq A$ and

$$
\phi_{i}=\frac{q_{i}}{r^{i}} \geq \frac{q_{i+1}}{r^{i}}=\phi_{i+1} r
$$

follow from $0 \leq q_{i} \leq A r^{i}$ and the monotonicity assumption on $\left\{q_{i}\right\}$, respectively.

If we can find a bound of the form $\left|h_{i}\right| \leq B_{i}$, for $i \geq 1$, then

$$
\left|\omega_{i}\right|=\left|h_{i}\right| r^{i} \leq B_{i} r^{i}
$$


Now, consider solving for the coefficients of $\Omega^{*}$ in terms of those of $Q^{*}$, that is, solving for $h_{i}$ in

$$
\begin{aligned}
1 & =Q^{*}(z) \Omega^{*}(z)=\left(1+\phi_{1} z+\phi_{2} z^{2}+\cdots\right)\left(1+h_{1} z+h_{2} z^{2}+\cdots\right) \\
& =1+\left(\phi_{1}+h_{1}\right) z+\left(\phi_{2}+\phi_{1} h_{1}+h_{2}\right) z^{2}+\left(\phi_{3}+\phi_{2} h_{1}+\phi_{1} h_{2}+h_{3}\right) z^{3}+\cdots .
\end{aligned}
$$

This yields the general linear recurrence

$$
\begin{aligned}
h_{1} & =-\phi_{1}, \\
h_{2} & =-\phi_{2}-\phi_{1} h_{1}, \\
h_{3} & =-\phi_{3}-\phi_{2} h_{1}-\phi_{1} h_{2}, \\
\vdots & \\
h_{n} & =-\phi_{n}-\phi_{n-1} h_{1}-\cdots-\phi_{1} h_{n-1},
\end{aligned}
$$

for each $n \in \mathbb{N}$.

To simplify the notation, we will represent the recurrence coefficients in (2.5) by the matrix

$$
\left[\begin{array}{cccc}
-\phi_{1} & 0 & \cdots & 0 \\
-\phi_{2} & -\phi_{1} & \ddots & \vdots \\
\vdots & \vdots & \ddots & 0 \\
-\phi_{n} & -\phi_{n-1} & \cdots & -\phi_{1}
\end{array}\right] .
$$

Now, by relaxing the Toeplitz restriction in (2.6), we can consider, instead, the matrix

$$
\left[\begin{array}{cccc}
-\alpha_{1,0} & 0 & \cdots & 0 \\
-\alpha_{2,0} & -\alpha_{2,1} & \ddots & \vdots \\
\vdots & \vdots & \ddots & 0 \\
-\alpha_{n, 0} & -\alpha_{n, 1} & \cdots & -\alpha_{n, n-1}
\end{array}\right] .
$$

The remaining restrictions on the $\left\{\phi_{i}\right\}$ imply

$$
0 \leq \alpha_{i, j} \leq A
$$

and (the quasimonotone restriction)

$$
\alpha_{i, j} \leq \alpha_{i, j+1} \frac{1}{r} .
$$

Define the sequence $\left\{B_{i}\right\}_{i=0}^{\infty}$ by the second-order recurrence

$$
B_{n}= \begin{cases}A^{n}, & \text { if } 0 \leq n \leq 2, \\ A B_{n-1}+(1-A r) B_{n-2}, & \text { if } n>2\end{cases}
$$

Note that $\left\{B_{i}\right\}$ is nondecreasing in $i$, under assumption (a) of Theorem 1.2. 
The key to obtaining a bound of the form in (2.3) will be the following lemma on bounds for linear recurrences, which will be proved in Section 4.

Lemma 2.1. Suppose the sequence $\left\{b_{n}\right\}_{n=0}^{\infty}$ satisfies $b_{0} \in\{-1,1\}$ and

$$
b_{n}=\sum_{k=0}^{n-1}\left(-\alpha_{n, k}\right) b_{k}, \quad n \geq 1,
$$

where

$$
\alpha_{n, k} \in[0, A]
$$

for $0 \leq k \leq n-1, n \geq 1$, and

$$
\alpha_{n, k} \leq \frac{1}{r} \alpha_{n, k+1}
$$

for $k \leq n-1, n \geq 2$. If $A$ and $r$ satisfy the hypotheses of Theorem 1.2, then

$$
\left|b_{n}\right| \leq B_{n}
$$

for $n \geq 0$.

Theorem 1.2 follows directly from Lemma 2.1, upon solving the second-order linear equation in (2.10) and employing (2.3).

Remark 2.2. Perhaps unexpectedly, Lemma 2.1 does not hold for all $A \geq 1$ and $r<1$. This fact is illustrated in the following simple example.

Example 2.3. Suppose that $b_{0}=1$, and $\left\{b_{i}\right\}$ and $\left\{\alpha_{i, j}\right\}$ satisfy (2.11), where the coefficient matrix in (2.7) is given by

$$
\left[-\alpha_{i, j}\right]=\left[\begin{array}{ccccccc}
-1 & 0 & 0 & 0 & 0 & 0 & 0 \\
-1 & -0.5 & 0 & 0 & 0 & 0 & 0 \\
0 & -1 & -1 & 0 & 0 & 0 & 0 \\
0 & -1 & -0.5 & -0.25 & 0 & 0 & 0 \\
-1 & -0.5 & -0.25 & -1 & -1 & 0 & 0 \\
-1 & -0.5 & -0.25 & -1 & -0.5 & -0.25 & 0 \\
0 & -1 & -0.5 & -0.25 & -0.125 & -1 & -1
\end{array}\right]
$$


Here, $\left(b_{0}, \ldots, b_{7}\right)=(1,-1,-0.5,1.5,0.875,-2.75,-1.625,5.140625)$. With $A=1$ and $r=.5,(2.12)$ and $(2.13)$ are satisfied, for $n \leq 7$, but $B_{7}=5.125<5.140625=b_{7}$. Hence, the inequality $b_{7} \leq B_{7}$ does not hold in this case. It may be easily verified that of the conditions (a)-(d) of Theorem 1.2 only the first is satisfied for $(A, r)=(1, .5)$.

Note that, recurrences with varying or random coefficients have been studied by many previous authors. For a partial survey of such literature see Viswanath [31, 32], Viswanath and Trefethen [33], Embree and Trefethen [13], Wright and Trefethen [35], Mallik [20], Popenda [25], Kittappa [18], Odlyzko [22], Berenhaut and Goedhart [8, 9], Berenhaut and Morton [10], Berenhaut and Foley [6], Zhang and Tian [37], Li and Cheng [19] and Stević [28-30] (and the references therein). For a comprehensive treatment of difference equations and inequalities; see Agarwal [1].

\section{Discretization}

In this section, we recall a discretization theorem from [7], which is crucial in proving Lemma 2.1. For completeness, we include a proof here.

First, for a given vector $\mathbf{u}=\left(u_{0}, u_{1}, u_{2}, \ldots, u_{k}\right)$ satisfying $u_{0} \geq 0, u_{i} \geq 1$ for $1 \leq i \leq k$ and

$$
\sum_{i} u_{i}=N
$$

define the vector $\mathbf{p}_{\mathbf{u}}$ via

$$
\mathbf{p}_{\mathbf{u}} \stackrel{\text { def }}{=} A(\overbrace{0,0, \ldots, 0}^{u_{0}} ; r^{0}, r^{1}, \ldots, r^{u_{1}-1} ; r^{0}, r^{1}, \ldots, r^{u_{2}-1} ; r^{0}, r^{1}, \ldots, r^{u_{k}-1}) .
$$

In addition, define the set of vectors

$$
\mathscr{P}_{N}=\left\{\mathbf{p}_{\mathbf{u}}: \mathbf{u} \text { satisfies }(3.1)\right\}
$$

We require the following lemma regarding inner products.

Lemma 3.1. Suppose that $\mathbf{p}=\left(p_{1}, \ldots, p_{n}\right)$ and $\mathbf{q}=\left(q_{1}, \ldots, q_{n}\right)$ are $n$-dimensional vectors where $\mathbf{p}$ satisfies

$$
r p_{i} \leq p_{i+1}
$$

for $1 \leq i \leq n-1$, and $0 \leq p_{i} \leq A$, for $1 \leq i \leq n$. Then

$$
\min \left\{\mathbf{p}_{\mathbf{u}} \cdot \mathbf{q}: \mathbf{p}_{\mathbf{u}} \in \mathscr{P}_{n}\right\} \leq \mathbf{p} \cdot \mathbf{q} \leq \max \left\{\mathbf{p}_{\mathbf{u}} \cdot \mathbf{q}: \mathbf{p}_{\mathbf{u}} \in \mathscr{P}_{n}\right\},
$$

where $\mathbf{p} \cdot \mathbf{q}$ denotes the standard scalar product $\sum_{i=1}^{n} p_{i} q_{i}$.

For a vector $\mathbf{p}=\left(p_{1}, p_{2}, \ldots, p_{n}\right)$, we will use the notation $\mathbf{p}^{i, j}$ to indicate the vector consisting of the $i$ th through $j$ th entries in $\mathbf{p}$, that is,

$$
\mathbf{p}^{i, j}=\left(p_{i}, p_{i+1}, \ldots, p_{j}\right)
$$


Proof of Lemma 3.1. First, suppose $\mathbf{p} \cdot \mathbf{q}>0$, and note that the lower bound in (3.5) follows from the fact that $\mathbf{p}_{\mathbf{u}}=\mathbf{0}$ for $\mathbf{u}=(n, 0, \ldots, 0)$. Now, consider the vectors $\widetilde{\mathbf{p}}_{i}=$ $\left(\widetilde{\mathbf{p}}_{i}(1), \widetilde{\mathbf{p}}_{i}(2), \ldots, \widetilde{\mathbf{p}}_{i}(n)\right), i=-1,0,1, \ldots, n-1$, defined recursively according to the following scheme.

(1) Set $\widetilde{\mathbf{p}}_{-1}=\mathbf{p}$.

(2) If $S_{i}=\left\{s: n-i+1 \leq s \leq n\right.$ and $\left.\widetilde{\mathbf{p}}_{i-1}(s)=A\right\}$ is a nonempty set, set $v_{i}=\min S_{i}$, otherwise set $v_{i}=n+1$.

(3) For $i \geq 0$, set

$$
\begin{aligned}
\widetilde{\mathbf{p}}_{i}= & \left(\widetilde{\mathbf{p}}_{i-1}(1), \widetilde{\mathbf{p}}_{i-1}(2), \ldots, \widetilde{\mathbf{p}}_{i-1}(n-i-1),\right. \\
& c_{i} \widetilde{\mathbf{p}}_{i-1}(n-i), c_{i} \widetilde{\mathbf{p}}_{i-1}(n-i+1), \ldots, c_{i} \widetilde{\mathbf{p}}_{i-1}\left(v_{i}-1\right), \\
& \left.\widetilde{\mathbf{p}}_{i-1}\left(v_{i}\right), \widetilde{\mathbf{p}}_{i-1}\left(v_{i}+1\right), \ldots, \widetilde{\mathbf{p}}_{i-1}(n)\right),
\end{aligned}
$$

where $c_{i}$ is given by

$$
c_{i}= \begin{cases}\frac{A}{\widetilde{\mathbf{p}}_{i-1}(n-i)}, & \text { if } \tilde{\mathbf{p}}_{i-1}^{n-i, v_{i}-1} \cdot \mathbf{q}^{n-i, v_{i}-1}>0, \\ \frac{r \widetilde{\mathbf{p}}_{i-1}(n-i-1)}{\widetilde{\mathbf{p}}_{i-1}(n-i)}, & \text { if } \widetilde{\mathbf{p}}_{i-1}^{n-i, v_{i}-1} \cdot \mathbf{q}^{n-i, v_{i}-1} \leq 0, i<n-1, \\ 0, & \text { otherwise. }\end{cases}
$$

Now, note that (3.7) and (3.8) imply that

$$
\widetilde{\mathbf{p}}_{i} \cdot \mathbf{q}-\widetilde{\mathbf{p}}_{i-1} \cdot \mathbf{q}=\left(c_{i}-1\right)\left(\tilde{\mathbf{p}}_{i-1}^{n-i, v_{i}-1} \cdot \mathbf{q}^{n-i, v_{i}-1}\right) \geq 0,
$$

and that if $\widetilde{\mathbf{p}}_{i-1}$ satisfies

$$
\widetilde{\mathbf{p}}_{i-1}(j-1) r \leq \widetilde{\mathbf{p}}_{i-1}(j) \leq A,
$$

then $\widetilde{\mathbf{p}}_{i}$ satisfies

$$
\widetilde{\mathbf{p}}_{i}(j-1) r \leq \widetilde{\mathbf{p}}_{i}(j) \leq A .
$$

It is not difficult to verify that the final $\widetilde{\mathbf{p}}_{i}$ (i.e., $\widetilde{\mathbf{p}}_{n-1}$ ) is of the form in (3.2), and the lemma is proven in this case. The proof follows similarly if $\mathbf{p} \cdot \mathbf{q} \leq 0$, and the proof of the lemma is complete.

We are now in a position to prove the following theorem from [7].

Theorem 3.2. Suppose that $\left\{b_{i}\right\}$ and $\left\{\alpha_{i, j}\right\}$ satisfy (2.11) and (2.13). Then, there exist $\left\{b_{i}^{\prime}\right\}$ and $\left\{\alpha_{i, j}^{\prime}\right\}$ such that

$$
\begin{gathered}
\left|b_{n}\right| \leq\left|b_{n}^{\prime}\right|, \\
b_{n}^{\prime}=\sum_{k=0}^{n-1}\left(-\alpha_{n, k}^{\prime}\right) b_{k}^{\prime}, \quad n \geq 1,
\end{gathered}
$$


Kenneth S. Berenhaut et al. 9

with each vector

$$
\boldsymbol{\alpha}_{i}^{\prime}=\left[\alpha_{i, 0}^{\prime}, \alpha_{i, 1}^{\prime}, \ldots, \alpha_{i, i-1}^{\prime}\right] \in \mathscr{P}_{i}
$$

for $1 \leq i \leq n$, where $\mathscr{P}_{i}$ is as in (3.3).

In fact, there exists a set $\left\{\boldsymbol{\alpha}_{1}^{\prime}, \boldsymbol{\alpha}_{2}^{\prime}, \ldots, \boldsymbol{\alpha}_{n}^{\prime}\right\}$, with $\boldsymbol{\alpha}_{i}^{\prime} \in \mathscr{P}_{i}$, such that $b_{i}^{\prime}$ is as large as possible (with its inherent sign) given $b_{0}, b_{1}^{\prime}, b_{2}^{\prime}, \ldots, b_{i-1}^{\prime}$.

Proof. The proof, here, involves applying Lemma 3.1 to successively "scale" the rows of the coefficient matrix $\left[-\alpha_{i, j}\right]$ as in (2.7), while not decreasing the value of $\left|b_{n}\right|$ at any step.

First, define the sequences

$$
\begin{gathered}
\overline{\boldsymbol{\alpha}}_{i}=\left(\alpha_{i, 0}, \ldots, \alpha_{i, i-1}\right), \\
\mathbf{b}^{k, j}=\left(b_{k}, \ldots, b_{j}\right),
\end{gathered}
$$

for $0 \leq k \leq j \leq n-1$ and $1 \leq i \leq n$.

Assume that $b_{n}>0$. Note that expanding via $(2.11), b_{n}$ can be written as

$$
b_{n}=C_{1}^{0} b_{0}+C_{1}^{1} b_{1}
$$

where $C_{1}^{0}$ and $C_{1}^{1}$ are constants, which depend on $\left\{\alpha_{i, j}\right\}$. If $C_{1}^{1}>0$, then select $\overline{\boldsymbol{\alpha}}_{1}^{\prime}=\left(\alpha_{1,0}^{\prime}\right) \in$ $\mathscr{P}_{1}$ so that $-\overline{\boldsymbol{\alpha}}_{1}^{\prime} \cdot \mathbf{b}^{0,0}$ is maximal, via Lemma 3.1. Similarly, if $C_{1}^{1}<0$, select $\overline{\boldsymbol{\alpha}}_{1}^{\prime}=\left(\alpha_{1,0}^{\prime}\right) \in$ $\mathscr{P}_{1}$ so that $-\overline{\boldsymbol{\alpha}}_{1}^{\prime} \cdot \mathbf{b}^{0,0}$ is minimal. In either case, replacing $\alpha_{1,0}$ by $\alpha_{1,0}^{\prime}$ in (2.11) will result in a larger (or equal) value for $C_{1}^{1} b_{1}$, and in turn, referring to (3.15), a larger (or equal) value of $\left|b_{n}\right|$.

Now, suppose that the first through $(k-1)$ th rows of the $\alpha$ matrix are of the form described in the theorem (i.e., resulting in maximal $b_{i}$ values for $1 \leq i \leq k-1$ with respect to the preceeding $\left.b_{j}, 0 \leq j \leq i-1\right)$, and express $b_{n}$ in the form

$$
b_{n}=C_{k}^{0} b_{0}+C_{k}^{1} b_{1}+\cdots+C_{k}^{k} b_{k}
$$

via (2.11). If $C_{k}^{k} \geq 0$, then select $\overline{\boldsymbol{\alpha}}_{k}^{\prime} \in \mathscr{P}_{1}$ so that $-\overline{\boldsymbol{\alpha}}_{k}^{\prime} \cdot \mathbf{b}^{0, k-1}$ is maximal, via Lemma 3.1. Similarly, if $C_{k}^{k}<0$, select $\overline{\boldsymbol{\alpha}}_{k}^{\prime} \in \mathscr{P}_{1}$ so that $-\overline{\boldsymbol{\alpha}}_{k}^{\prime} \cdot \mathbf{b}^{0,0}$ is minimal. In either case, referring to (3.16), replacing the values in $\overline{\boldsymbol{\alpha}}_{k}$ by those in $\overline{\boldsymbol{\alpha}}_{k}^{\prime}$ in (2.11) will not decrease the value of $\left|b_{n}\right|$. The result follows similarly for the case $C_{k}^{k}<0$, and the theorem follows by induction for this case. The case $b_{n} \leq 0$ is similar and the theorem is proven.

Remark 3.3. A simpler version of Theorem 3.2 was also employed in Berenhaut and Bandyopadhyay [5] in proving that all symmetric Toeplitz matrices generated by monotone convex sequences have off-diagonal decay preserved through triangular decompositions.

A matrix of coefficients will be said to be "fully scaled" when the process suggested in the proof of Theorem 1.1 terminates; that is, the set $\left\{\boldsymbol{\alpha}_{1}^{\prime}, \boldsymbol{\alpha}_{2}^{\prime}, \ldots, \boldsymbol{\alpha}_{n}^{\prime}\right\}$ referred to in the last sentence of the statement of Theorem 1.1 is attained.

We now turn to a proof of Lemma 2.1. 


\section{Proof of Lemma 2.1}

By Theorem 3.2, we may restrict attention to sequences $\left\{b_{i}\right\}$ resulting from the "fully scaled" matrices of $\alpha_{i, j}$. Hence, suppose we have some "fully scaled" matrix [- $\left.\alpha_{i, j}\right]$, where the resulting $\left\{b_{i}\right\}_{i=0}^{n}$ has sign configuration $\mathbf{s}=\left\{s_{i}\right\}$, that is,

$$
s_{i}= \begin{cases}+1, & \text { if } b_{i} \geq 0 \\ -1, & \text { if } b_{i}<0\end{cases}
$$

The following lemma is a direct consequence of Theorem 3.2, via the fact that the $\alpha$ matrix is fully scaled, and will be used frequently, without explicit mention, in what follows.

Lemma 4.1. Suppose that $\left[\alpha_{i, j}\right]$ is fully scaled. Then,

(i) if $k<K$ and $s_{k}=s_{K}$, then

$$
s_{k}\left(\sum_{i=0}^{k-1}\left(-\alpha_{K, i}\right) b_{i}\right) \leq s_{k} b_{k}
$$

(ii) if $s_{k}=s_{k-1}$, then $s_{k} b_{k} \leq s_{k} b_{k-1}$,

(iii) if $k<K$ and $s_{K}=s_{k} \neq s_{k-1}$, then

$$
s_{k}\left(\sum_{i=0}^{k-2}\left(-\alpha_{K, i}\right) b_{i}\right) \leq s_{k}\left(b_{k}+A b_{k-1}\right)
$$

Proof. Without loss of generality, we may assume that $s_{k}=1$. For part (i), we have

$$
b_{k}=\sum_{i=0}^{k-1}\left(-\alpha_{k, i}\right) b_{i} \geq \sum_{i=0}^{k-1}\left(-\alpha_{K, i}\right) b_{i}
$$

since $\left[\alpha_{i, j}\right]$ is fully scaled. Similarly, for (ii), we have

$$
b_{k}=\sum_{i=0}^{k-2}\left(-\alpha_{k, i}\right) b_{i}-\alpha_{k, k-1} b_{k-1} \leq \sum_{i=0}^{k-2}\left(-\alpha_{k, i}\right) b_{i} \leq \sum_{i=0}^{k-2}\left(-\alpha_{k-1, i}\right) b_{i}=b_{k-1} .
$$

To prove (iii), note that $\alpha_{k, k-1}=A$ and hence

$$
b_{k}+A b_{k-1}=\sum_{i=0}^{k-2}\left(-\alpha_{k, i}\right) b_{i} \geq \sum_{i=0}^{k-2}\left(-\alpha_{K, i}\right) b_{i}
$$

The next technical lemma is proved in Section 5. 
Lemma 4.2. Suppose that $A$ and $r$ satisfy the hypotheses of Theorem 1.2, $c, k \geq 2$, and $n \geq$ $\max \{c+k, c+3\}$. Then,

$$
\left((c-1) A^{2}+1-A\left(r^{k}+\cdots+r^{k+c-2}\right)\right) B_{n-c}+A r^{k-1} B_{n-c-2} \leq B_{n} .
$$

Proof. See Section 5.

We now turn to a proof of Lemma 2.1.

Proof of Lemma 2.1. Suppose we have some "fully scaled" matrix of $\alpha_{i, j}$ where the resulting $\left\{b_{i}\right\}$ has sign configuration $\mathbf{s}=\left\{s_{i}\right\}$.

By employing Theorem 3.2 and comparing several low degree polynomials in $A$ and $r$, it is not difficult to verify that

$$
\left|b_{i}\right| \leq B_{i}
$$

for $i \leq 3$. Now, assume that $\left|b_{i}\right| \leq B_{i}$ for all $i \leq n-1$.

Note that by Theorem 3.2, if for some $j<i, s_{i}=s_{j}\left(s_{i} \neq s_{j}\right)$, then we need only consider $\alpha_{i, j}$ of the form $\alpha_{i, j}=A r^{k}$ for some $k \geq 1(k \geq 0)$.

We will consider the following cases:

(1) $s_{n-1}=s_{n}=1$;

(2) $s_{n-2}=s_{n-1}=-1, s_{n}=1$;

(3) $s_{n}=1, s_{n-1}=-1, s_{n-2}=s_{n-3}=\cdots=s_{n-c}=1, s_{n-c-1}=-1, \alpha_{n, n-c}=A r$;

(4) $s_{n}=1, s_{n-1}=-1, s_{n-2}=s_{n-3}=\cdots=s_{n-c}=1, s_{n-c-1}=-1, \alpha_{n, n-c}=A r^{k}$ for some $k \geq 2$ and

(a) $s_{n-c-2}=1$;

(b) $s_{n-c-2}=-1$.

The proofs for the cases where the signs are opposite to those considered are analogous.

Case $1\left(s_{n-1}=s_{n}=1\right.$, i.e., $\left.\mathbf{s}=(\ldots, 1,1)\right)$. Here,

$$
b_{n}=-\alpha_{n, n-1} b_{n-1}+\sum_{i=0}^{n-2}\left(-\alpha_{n, i}\right) b_{i} \leq-\alpha_{n, n-1} b_{n-1}+B_{n-1} \leq B_{n-1} \leq B_{n} .
$$

Case $2\left(s_{n-2}=s_{n-1}=-1, s_{n}=1\right.$, i.e., $\left.\mathbf{s}=(\ldots,-1,-1,1)\right)$. Note that $\alpha_{n, n-1}=\alpha_{n, n-2}=A$, and consider the $\alpha$-matrix obtained by switching the positions of $\alpha_{n, i}$ and $\alpha_{n-1, i}$ for $0 \leq$ $i \leq n-2$. Denote the resulting $b$-vector by $\mathbf{b}^{*}=\left(b_{1}^{*}, b_{2}^{*}, \ldots, b_{n}^{*}\right)$. Then,

$$
b_{n-1}^{*}=b_{n}+A b_{n-1},
$$

and hence

$$
\begin{aligned}
b_{n}^{*}+b_{n} & =-A b_{n-1}^{*}+b_{n-1}+b_{n}=-A\left(b_{n}+A b_{n-1}\right)+b_{n-1}+b_{n} \\
& =(1-A) b_{n}+\left(1-A^{2}\right) b_{n-1}=(1-A)\left(b_{n}+(1+A) b_{n-1}\right) .
\end{aligned}
$$


Setting $\alpha_{n, 0}=\alpha_{n, 1}=\cdots=\alpha_{n, n-2}=0$ shows that $b_{n} \geq-A b_{n-1}-A b_{n-2}$, hence

$$
b_{n}+(1+A) b_{n-1} \geq-A b_{n-2}+b_{n-1} \geq 0
$$

since $b_{n-2} \leq b_{n-1} \leq 0$ (by Lemma 4.1 (ii)) and $A \geq 1$.

Since $b_{n-1}^{*} \geq 0, b_{n}^{*}<0$, and $\left|b_{n}^{*}\right| \geq\left|b_{n}\right|$, we have reduced this case to one of Cases 3 or 4 .

For the remaining cases, it will be useful to introduce the following notation (similar to (3.6)) for subsequences of recurrence coefficients.

Definition 4.3. For $0 \leq k \leq j \leq i-1$ and $i \leq n$, define the subsequences

$$
\overline{\boldsymbol{\alpha}}_{i}^{k, j} \stackrel{\text { def }}{=}\left[-\alpha_{i, k}, \ldots,-\alpha_{i, j}\right]
$$

Case $3\left((\mathrm{i}) s_{n}=1, s_{n-1}=-1, s_{n-2}=s_{n-3}=\cdots=s_{n-c}=1, s_{n-c-1}=-1\right.$, i.e., $\mathbf{s}=(\ldots,-1$, $\underbrace{1, \ldots, 1}_{c-1},-1,1)$, and (ii) $\alpha_{n, n-c}=A r$ ).

Theorem 3.2 leads to the following entries in the $\alpha$-matrix:

$$
\begin{gathered}
\overline{\boldsymbol{\alpha}}_{n}^{n-c-1, n-1}=-A\left[1, r^{1}, r^{2}, \ldots, r^{k+c-2}, 1\right], \\
\overline{\boldsymbol{\alpha}}_{n-c}^{n-c-1, n-c-1}=-A[1] .
\end{gathered}
$$

We have

$$
b_{n}=-A b_{n-1}+\sum_{j=n-c+1}^{n-2}-A r^{j-(n-c)+1} b_{j}-A r b_{n-c}+\sum_{j=0}^{n-c-1}-\alpha_{n, j} b_{j} .
$$

Since $b_{j} \geq 0$ for $n-c+1 \leq j \leq n-2$, the first sum in (4.15) is nonpositive, while by Lemma 4.1(i), the second sum is bounded by $b_{n-c}$. Thus, we have

$$
\begin{aligned}
b_{n} & \leq A B_{n-1}-A r b_{n-c}+b_{n-c}=A B_{n-1}+(1-A r) b_{n-c} \\
& \leq A B_{n-1}+(1-A r) B_{n-c} \leq A B_{n-1}+(1-A r) B_{n-2}=B_{n} .
\end{aligned}
$$

Case 4.

Subcase 4.1 ((i) $s_{n}=1, s_{n-1}=-1, s_{n-2}=s_{n-3}=\cdots=s_{n-c}=1, s_{n-c-1}=-1, s_{n-c-2}=1$, i.e., $\mathbf{s}=(\ldots, 1,-1, \underbrace{1, \ldots, 1}_{c-1},-1,1)$, and (ii) $\alpha_{n, n-c}=A r^{k}$ for some $\left.k \geq 2\right)$. Theorem 3.2 leads to the following entries in the $\alpha$-matrix:

$$
\begin{aligned}
\overline{\boldsymbol{\alpha}}_{n}^{n-c-1, n-1} & =-A\left[r^{k-1}, r^{k}, \ldots, r^{k+c-2}, 1\right], \\
\overline{\boldsymbol{\alpha}}_{n-1}^{n-c-2, n-2} & =-A\left[r^{\nu-1}, r^{v}, 1,1, \ldots, 1\right], \\
\overline{\boldsymbol{\alpha}}_{n-c}^{n-c-1, n-c-1} & =-A[1], \\
\overline{\boldsymbol{\alpha}}_{n-c-1}^{n-c-2, n-c-2} & =-A[1],
\end{aligned}
$$

for some $v \geq 1$. 
Employing (4.17) and applying Lemma 4.1(iii) give

$$
\begin{aligned}
b_{n} & =-A b_{n-1}+\sum_{j=n-c}^{n-2}-A r^{j-(n-c)+k} b_{j}-A r^{k-1} b_{n-c-1}+\sum_{j=0}^{n-c-2}-\alpha_{n, j} b_{j} \\
& \leq-A b_{n-1}+\sum_{j=n-c}^{n-2}-A r^{j-(n-c)+k} b_{j}-A r^{k-1} b_{n-c-1}+\left(b_{n-c}+A b_{n-c-1}\right), \\
b_{n-1} & =\sum_{j=n-c}^{n-2}(-A) b_{j}-A r^{v} b_{n-c-1}-A r^{\nu-1} b_{n-c-2}+\sum_{j=0}^{n-c-3}-\alpha_{n, j} b_{j} \\
& \geq \sum_{j=n-c}^{n-2}(-A) b_{j}-A r^{v} b_{n-c-1}-A r^{\nu-1} b_{n-c-2}+\left(b_{n-c-1}+A b_{n-c-2}\right) .
\end{aligned}
$$

Hence, substituting (4.19) into (4.18) and using Lemma 4.1(ii) to obtain $0 \leq b_{j} \leq b_{n-c} \leq$ $B_{n-c}$, for $n-c \leq j \leq n-2$, gives

$$
\begin{aligned}
b_{n} \leq & \sum_{j=n-c}^{n-2}\left(A^{2}-A r^{j-(n-c)+k}\right) b_{j}+b_{n-c}+\left(A-A r^{k-1}+A^{2} r^{v}-A\right) b_{n-c-1} \\
& +\left(A^{2} r^{v-1}-A^{2}\right) b_{n-c-2} \\
\leq & \sum_{j=n-c}^{n-2}\left(A^{2}-A r^{j-(n-c)+k}\right) B_{n-c}+B_{n-c}+\left(-A r^{k-1}+A^{2} r^{v}\right) b_{n-c-1} \\
& +\left(A^{2} r^{v-1}-A^{2}\right) b_{n-c-2} \\
= & \left((c-1) A^{2}+1-A\left(r^{k}+r^{k+1}+\cdots+r^{k+c-2}\right)\right) B_{n-c} \\
& +\left(A^{2} r^{v}-A r^{k-1}\right) b_{n-c-1}+\left(A^{2} r^{\nu-1}-A^{2}\right) b_{n-c-2} .
\end{aligned}
$$

Now, suppose $A^{2} r^{v}-A r^{k-1} \geq 0$. Then, since $b_{n-c-1}<0, b_{n-c-2} \geq 0$, and $A^{2}>A^{2} r^{v-1}$, (4.20) and Lemma 4.2 give

$$
b_{n} \leq\left((c-1) A^{2}+1-A\left(r^{k}+r^{k+1}+\cdots+r^{k+c-2}\right)\right) B_{n-c} \leq B_{n} .
$$

On the other hand, if $A^{2} r^{v}-A r^{k-1}<0$, then $1 \leq A<r^{k-1-v}$, and hence

$$
v>k-1 \geq 1 \text {. }
$$

Since

$$
0>b_{n-c-1}=-A b_{n-c-2}+\sum_{j=0}^{n-c-3}-\alpha_{n-c-1, j} b_{j} \geq-A b_{n-c-2}-B_{n-c-2}
$$


14 Reciprocals of formal power series

we have

$$
\begin{aligned}
\left(A^{2} r^{v}\right. & \left.-A r^{k-1}\right) b_{n-c-1}+\left(A^{2} r^{v-1}-A^{2}\right) b_{n-c-2} \\
& \leq\left(A^{2} r^{v}-A r^{k-1}\right)\left(-A b_{n-c-2}-B_{n-c-2}\right)+\left(A^{2} r^{\nu-1}-A^{2}\right) b_{n-c-2} \\
& =\left(-A^{3} r^{v}+A^{2} r^{k-1}+A^{2} r^{\nu-1}-A^{2}\right) b_{n-c-2}+\left(-A^{2} r^{v}+A r^{k-1}\right) B_{n-c-2} \\
& \leq A r^{k-1} B_{n-c-2} .
\end{aligned}
$$

The final inequality in (4.24) follows since $b_{n-c-2} \geq 0, v>1,0<r<1 / 2,0 \leq A r \leq 1$, and

$$
\begin{aligned}
-A^{3} r^{v}+A^{2} r^{k-1}+A^{2} r^{\nu-1}-A^{2} & =A^{2}\left(r^{\nu-1}(1-A r)-\left(1-r^{k-1}\right)\right) \\
& \leq A^{2}\left(r(1-A r)-\left(1-r^{k-1}\right)\right) \\
& \leq A^{2}(r-(1-r))=A^{2}(2 r-1) \leq 0 .
\end{aligned}
$$

Application of Lemma 4.2 to (4.20) and (4.24) completes the proof for this case.

Subcase 4.2 ((i) $s_{n}=1, s_{n-1}=-1, s_{n-2}=s_{n-3}=\cdots=s_{n-c}=1, s_{n-c-1}=-1, s_{n-c-2}=-1$, i.e., $\mathbf{s}=(\ldots,-1,-1, \underbrace{1, \ldots, 1}_{c-1},-1,1)$, and (ii) $\alpha_{n, n-c}=A r^{k}$ for some $\left.k \geq 2\right)$.

Theorem 3.2 leads to the following entries in the $\alpha$-matrix:

$$
\begin{aligned}
\overline{\boldsymbol{\alpha}}_{n}^{n-c-1, n-1} & =-A\left[r^{k-1}, r^{k}, \ldots, r^{k+c-2}, 1\right], \\
\overline{\boldsymbol{\alpha}}_{n-1}^{n-c-2, n-2} & =-A\left[r^{\nu-1}, r^{v}, 1,1, \ldots, 1\right], \\
\overline{\boldsymbol{\alpha}}_{n-c}^{n-c-2, n-c-1} & =-A[1,1],
\end{aligned}
$$

for some $v \geq 1$.

Employing Lemma 4.1(i) and (iii), we have

$$
\begin{aligned}
b_{n} & =-A b_{n-1}+\sum_{j=n-c}^{n-2}-A r^{j-(n-c)+k} b_{j}-A r^{k-1} b_{n-c-1}+\sum_{j=0}^{n-c-2}-\alpha_{n, j} b_{j} \\
& \leq-A b_{n-1}+\sum_{j=n-c}^{n-2}-A r^{j-(n-c)+k} b_{j}-A r^{k-1} b_{n-c-1}+\left(b_{n-c}+A b_{n-c-1}\right), \\
b_{n-1} & =\sum_{j=n-c}^{n-2}(-A) b_{j}-A r^{v} b_{n-c-1}+\sum_{j=0}^{n-c-2}-\alpha_{n, j} b_{j} \\
& \geq \sum_{j=n-c}^{n-2}(-A) b_{j}-A r^{v} b_{n-c-1}+b_{n-c-1} .
\end{aligned}
$$


Hence, substituting (4.28) into (4.27) gives

$$
\begin{aligned}
b_{n} & \leq \sum_{j=n-c}^{n-2}\left(A^{2}-A r^{j-(n-c)+k}\right) b_{j}+b_{n-c}+\left(A-A r^{k-1}+A^{2} r^{v}-A\right) b_{n-c-1} \\
& \leq \sum_{j=n-c}^{n-2}\left(A^{2}-A r^{j-(n-c)+k}\right) B_{n-c}+B_{n-c}+\left(-A r^{k-1}+A^{2} r^{v}\right) b_{n-c-1} \\
& =\left((c-1) A^{2}+1-A\left(r^{k}+r^{k+1}+\cdots+r^{l}\right)\right) B_{n-c}+\left(A^{2} r^{v}-A r^{k-1}\right) b_{n-c-1} \\
& \leq\left((c-1) A^{2}+1-A\left(r^{k}+r^{k+1}+\cdots+r^{l}\right)\right) B_{n-c}+A r^{k-1} b_{n-c-2} \\
& \leq\left((c-1) A^{2}+1-A\left(r^{k}+r^{k+1}+\cdots+r^{l}\right)\right) B_{n-c}+A r^{k-1} B_{n-c-2},
\end{aligned}
$$

where $l=k+c-2$, and the result for this case follows by Lemma 4.2.

\section{Proof of Lemma 4.2}

In this section, we will prove Lemma 4.2.

First, note that straightforward manipulation of (2.10), along with the fact that $\left\{B_{i}\right\}$ is nondecreasing, gives $B_{i} \leq(A+1-A r) B_{i-1}$ for all $i \geq 3$, and hence

$$
\left(A+\frac{1-A r}{A+(1-A r)}\right) B_{n-1} \leq B_{n} \leq(A+1-A r) B_{n-1},
$$

for $n \geq 3$.

Proof of Lemma 4.2. We will consider two cases: (1) $c=2$ and (2) $c \geq 3$.

Case $1(c=2)$. Set

$$
Z \stackrel{\text { def }}{=}\left(A^{2}+1-A r^{k}\right) B_{n-2}+A r^{k-1} B_{n-4}
$$

We have

$$
\begin{aligned}
B_{n}-Z & =A B_{n-1}+(1-A r) B_{n-2}-Z \\
& =A\left(A B_{n-2}+(1-A r) B_{n-3}\right)+(1-A r) B_{n-2}-Z \\
& =\left(A r^{k}-A r\right) B_{n-2}+A(1-A r) B_{n-3}-A r^{k-1} B_{n-4} \\
& =\left(A r^{k}-A r\right)\left(A B_{n-3}+(1-A r) B_{n-4}\right)+A(1-A r) B_{n-3}-A r^{k-1} B_{n-4} \\
& =\left(A^{2} r^{k}-2 A^{2} r+A\right) B_{n-3}+\left(\left(A r^{k}-A r\right)(1-A r)-A r^{k-1}\right) B_{n-4} \\
& =A\left(\kappa_{1} B_{n-3}+\kappa_{2} B_{n-4}\right),
\end{aligned}
$$

for instance. Since $A r<1 / 2, \kappa_{1}=1-A\left(2 r-r^{k}\right)>0$, and hence, applying (5.1), (5.3), and assumption (a) of Theorem 1.2 gives

$$
\begin{aligned}
B_{n}-Z & \geq \kappa_{1} B_{n-3}+\kappa_{2} B_{n-4} \geq\left(\kappa_{1}\left(A+\frac{1-A r}{A+1-A r}\right)+\kappa_{2}\right) B_{n-4} \\
& \geq\left(\kappa_{1}\left(A+\frac{1 / 2}{A+1 / 2}\right)+\kappa_{2}\right) B_{n-4} .
\end{aligned}
$$


Now, define $T$ by

$$
T(k) \stackrel{\text { def }}{=} \kappa_{1}\left(A+\frac{1-A r}{A+1-A r}\right)+\kappa_{2} .
$$

Then,

$$
\begin{aligned}
T^{\prime}(k) & =A^{2} r \ln (r)\left(A+\frac{1-A r}{A+1-A r}\right)+A r^{k} \ln (r)-A^{2} r^{k+1} \ln (r)-A r^{k-1} \ln (r) \\
& =r^{k-1}\left(\frac{A \ln (r)\left(A^{3} r+A^{2} r-A^{3} r^{2}+3 A r-2 A^{2} r^{2}+r-2 A r^{2}+A^{2} r^{3}-A-1\right)}{A+1-A r}\right) .
\end{aligned}
$$

Hence, for fixed $(A, r), T$ is monotone in $k$ and we need only consider two cases: $T(2)$ and $\lim _{k \rightarrow \infty} T(k)$. Assumption (b) in the statement of Theorem 1.2 (with (5.4)) gives $T(2) \geq$ 0 , while assumption (c) gives $\lim _{k \rightarrow \infty} T(k) \geq 0$, and the lemma follows for this case.

Case $2(c \geq 3)$. First, note that

$$
\begin{aligned}
&\left(A^{2}(c-1)+1-A\left(r^{k}+\cdots+r^{k+c-2}\right)\right) B_{n-c}+A r^{k-1} B_{n-c-2} \\
&=\left(A^{2}(c-2)+1-A\left(r^{k}+\cdots+r^{k+c-3}\right)\right) B_{n-c} \\
&+A r^{k-1} B_{n-c-2}+A^{2} B_{n-c}-A r^{k+c-2} B_{n-c} \\
&=\left(A^{2}(c-2)+1-A\left(r^{k}+\cdots+r^{k+(c-1)-2}\right)\right) B_{(n-1)-(c-1)} \\
&+A r^{k-1} B_{(n-1)-(c-1)-2}+A^{2} B_{n-c}-A r^{k+c-2} B_{n-c} \\
& \leq B_{n-1}+A^{2} B_{n-c} \leq B_{n-1}+A^{2} B_{n-3}
\end{aligned}
$$

by induction.

Now, let

$$
W \stackrel{\text { def }}{=} B_{n}-\left(B_{n-1}+A^{2} B_{n-3}\right)
$$

Successively employing (2.10) gives

$$
\begin{aligned}
W & =A B_{n-1}+(1-A r) B_{n-2}-\left(B_{n-1}+A^{2} B_{n-3}\right) \\
& =(A-1)\left(A B_{n-2}+(1-A r) B_{n-3}\right)+(1-A r) B_{n-2}-A^{2} B_{n-3} \\
& =(A(A-1)+1-A r) B_{n-2}+\left((A-1)(1-A r)-A^{2}\right) B_{n-3} \\
& =\gamma_{1} B_{n-2}+\gamma_{2} B_{n-3}=\left(A \gamma_{1}+\gamma_{2}\right) B_{n-3}+\left(\gamma_{1}(1-A r)\right) B_{n-4},
\end{aligned}
$$

where $\gamma_{1} \stackrel{\text { def }}{=} A(A-1)+1-A r$ and $\gamma_{2} \stackrel{\text { def }}{=}(A-1)(1-A r)-A^{2}$. Since $\gamma_{1}>0$, we have (via (5.1)) that

$$
W \geq\left(\left(A \gamma_{1}+\gamma_{2}\right)+\left(\gamma_{1}(1-A r)\right) \frac{1}{A+1-A r}\right) B_{n-3} \geq 0
$$

by assumption (d) in the statement of Theorem 1.2.

Lemma 4.2 now follows. 


\section{Acknowledgment}

We are very thankful to the referee for comments and insights that substantially improved this manuscript.

\section{References}

[1] R. P. Agarwal, Difference Equations and Inequalities. Theory, Methods, and Applications, 2nd ed., Monographs and Textbooks in Pure and Applied Mathematics, vol. 228, Marcel Dekker, New York, 2000.

[2] P. H. Baxendale, Renewal theory and computable convergence rates for geometrically ergodic Markov chains, The Annals of Applied Probability 15 (2005), no. 1B, 700-738.

[3] F. Beaucoup, P. Borwein, D. W. Boyd, and C. Pinner, Multiple roots of $[-1,1]$ power series, Journal of the London Mathematical Society. Second Series 57 (1998), no. 1, 135-147.

[4] _ Power series with restricted coefficients and a root on a given ray, Mathematics of Computation 67 (1998), no. 222, 715-736.

[5] K. S. Berenhaut and D. Bandyopadhyay, Monotone convex sequences and Cholesky decomposition of symmetric Toeplitz matrices, Linear Algebra and Its Applications 403 (2005), 75-85.

[6] K. S. Berenhaut and J. D. Foley, Explicit bounds for multi-dimensional linear recurrences with restricted coefficients, Journal of Mathematical Analysis and Applications 322 (2006), no. 2, 11591167.

[7] K. S. Berenhaut, J. D. Foley, and D. Bandyopadhyay, Maximization for inner products under quasimonotone constraints, Journal of Inequalities in Pure and Applied Mathematics 7 (2006), no. 5, $1-23$.

[8] K. S. Berenhaut and E. G. Goedhart, Explicit bounds for second-order difference equations and a solution to a question of Stević, Journal of Mathematical Analysis and Applications 305 (2005), no. $1,1-10$.

[9] Second-order linear recurrences with restricted coefficients and the constant $(1 / 3)^{1 / 3}$, Mathematical Inequalities \& Applications 9 (2006), no. 3, 445-452.

[10] K. S. Berenhaut and D. C. Morton, Second-order bounds for linear recurrences with negative coefficients, Journal of Computational and Applied Mathematics 186 (2006), no. 2, 504-522.

[11] P. Borwein and T. Erdélyi, On the zeros of polynomials with restricted coefficients, Illinois Journal of Mathematics 41 (1997), no. 4, 667-675.

[12] P. Borwein and C. Pinner, Polynomials with $\{0,+1,-1\}$ coefficients and a root close to a given point, Canadian Journal of Mathematics 49 (1997), no. 5, 887-915.

[13] M. Embree and L. N. Trefethen, Growth and decay of random Fibonacci sequences, Proceedings of the Royal Society of London. Series A. Mathematical, Physical and Engineering Sciences $\mathbf{4 5 5}$ (1999), no. 1987, 2471-2485.

[14] W. Feller, An Introduction to Probability Theory and Its Applications. Vol. I, 3rd ed., John Wiley \& Sons, New York, 1968.

[15] L. Flatto, J. C. Lagarias, and B. Poonen, The zeta function of the beta transformation, Ergodic Theory and Dynamical Systems 14 (1994), no. 2, 237-266.

[16] C. R. Heathcote, Complete exponential convergence and some related topics, Journal of Applied Probability 4 (1967), no. 2, 217-256.

[17] D. G. Kendall, Unitary dilations of Markov transition operators, and the corresponding integral representations for transition-probability matrices, Probability and Statistics: The Harald Cramér Volume (U. Grenander, ed.), Almqvist \& Wiksell, Stockholm; John Wiley \& Sons, New York, 1959, pp. 139-161.

[18] R. K. Kittappa, A representation of the solution of the nth order linear difference equation with variable coefficients, Linear Algebra and Its Applications 193 (1993), 211-222. 
[19] W.-T. Li and S.-S. Cheng, Remarks on two recent oscillation theorems for second-order linear difference equations, Applied Mathematics Letters 16 (2003), no. 2, 161-163.

[20] R. K. Mallik, On the solution of a linear homogeneous difference equation with variable coefficients, SIAM Journal on Mathematical Analysis 31 (2000), no. 2, 375-385.

[21] S. P. Meyn and R. L. Tweedie, Markov Chains and Stochastic Stability, Communications and Control Engineering Series, Springer, London, 1993.

[22] A. M. Odlyzko, Asymptotic enumeration methods, Handbook of Combinatorics, Vol. 1, 2 (R. Graham, M. Groetschel, and L. Lovasz, eds.), Elsevier, Amsterdam, 1995, pp. 1063-1229.

[23] A. M. Odlyzko and B. Poonen, Zeros of polynomials with 0,1 coefficients, L'Enseignement Mathématique 39 (1993), no. 3-4, 317-348.

[24] C. Pinner, Double roots of $[-1,1]$ power series and related matters, Mathematics of Computation 68 (1999), no. 227, 1149-1178.

[25] J. Popenda, One expression for the solutions of second order difference equations, Proceedings of the American Mathematical Society 100 (1987), no. 1, 87-93.

[26] B. Solomyak, Conjugates of beta-numbers and the zero-free domain for a class of analytic functions, Proceedings of the London Mathematical Society. Third Series 68 (1994), no. 3, 477-498.

[27] F. M. Spieksma, Spectral conditions and bounds for the rate of convergence of countable Markov chains, Tech. Rep., University of Leiden, Leiden, 1993.

[28] S. Stević, Growth theorems for homogeneous second-order difference equations, The ANZIAM Journal 43 (2002), no. 4, 559-566.

[29] _ Asymptotic behaviour of second-order difference equations, The ANZIAM Journal 46 (2004), no. 1, 157-170.

[30] _ Growth estimates for solutions of nonlinear second-order difference equations, The ANZIAM Journal 46 (2005), no. 3, 439-448.

[31] D. Viswanath, Lyapunov exponents from random Fibonacci sequences to the Lorenz equations, Ph.D. thesis, Department of Computer Science, Cornell University, New York, 1998.

[32] _ Random Fibonacci sequences and the number 1.13198824..., Mathematics of Computation 69 (2000), no. 231, 1131-1155.

[33] D. Viswanath and L. N. Trefethen, Condition numbers of random triangular matrices, SIAM Journal on Matrix Analysis and Applications 19 (1998), no. 2, 564-581.

[34] H. S. Wilf, Generatingfunctionology, 2nd ed., Academic Press, Massachusetts, 1994.

[35] T. G. Wright and L. N. Trefethen, Computing Lyapunov constants for random recurrences with smooth coefficients, Journal of Computational and Applied Mathematics 132 (2001), no. 2, 331340.

[36] O. Yamamoto, On some bounds for zeros of norm-bounded polynomials, Journal of Symbolic Computation 18 (1994), no. 5, 403-427.

[37] B. G. Zhang and C. J. Tian, Some remarks on second order linear difference equations, Discrete Dynamics in Nature and Society 2 (1998), no. 2, 93-97.

Kenneth S. Berenhaut: Department of Mathematics, Wake Forest University, Winston-Salem,

NC 27109, USA

E-mail address: berenhks@wfu.edu

Edward E. Allen: Department of Mathematics, Wake Forest University, Winston-Salem,

NC 27109, USA

E-mail address: allene@wfu.edu

Sam J. Fraser: Department of Mathematics, Wake Forest University, Winston-Salem,

NC 27109, USA

E-mail address: sam-fraser@uiowa.edu 


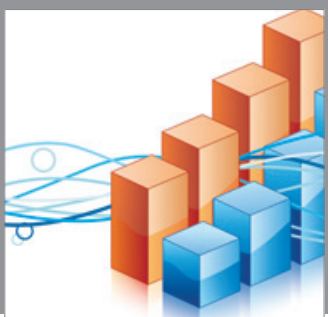

Advances in

Operations Research



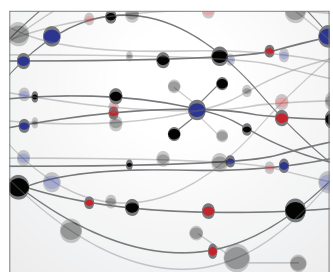

\section{The Scientific} World Journal
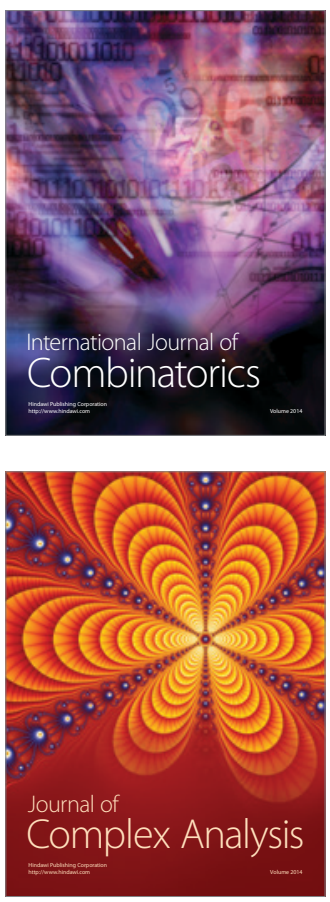

International Journal of

Mathematics and

Mathematical

Sciences
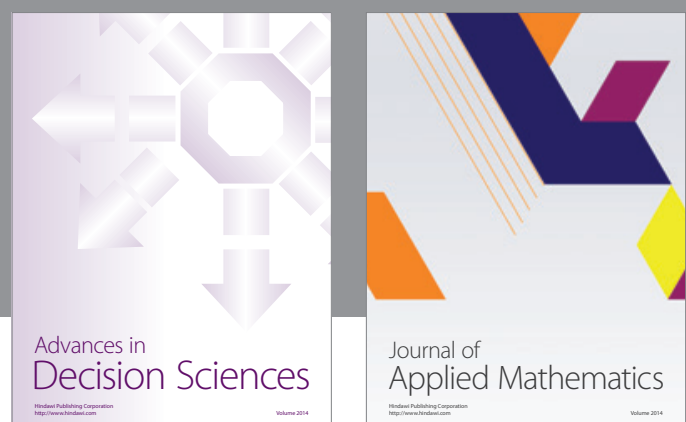

Journal of

Applied Mathematics
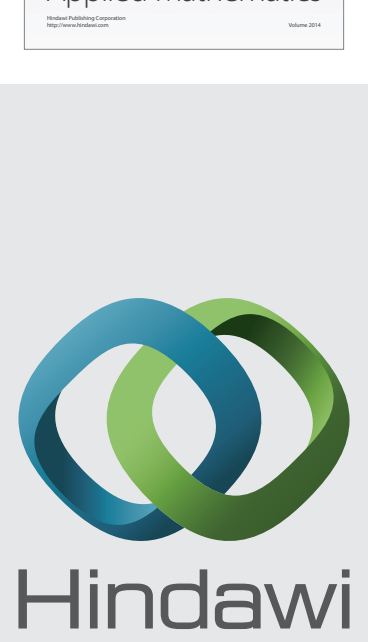

Submit your manuscripts at http://www.hindawi.com
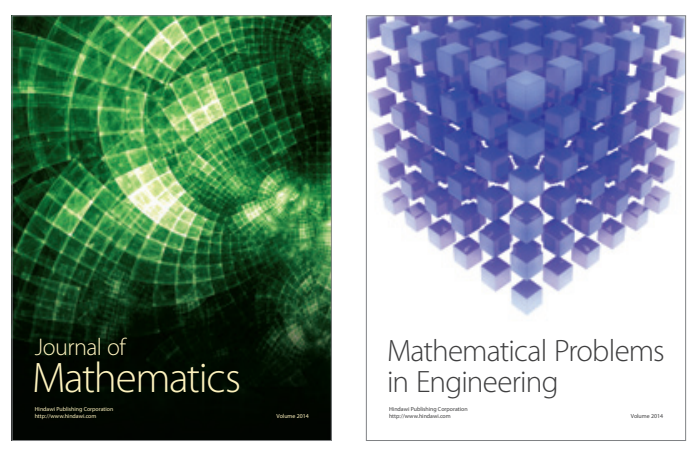

Mathematical Problems in Engineering
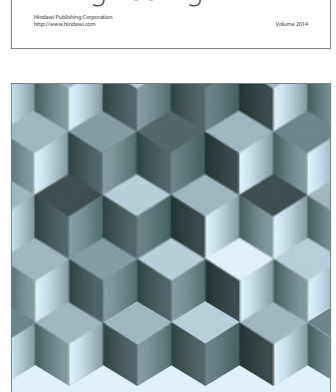

Journal of

Function Spaces
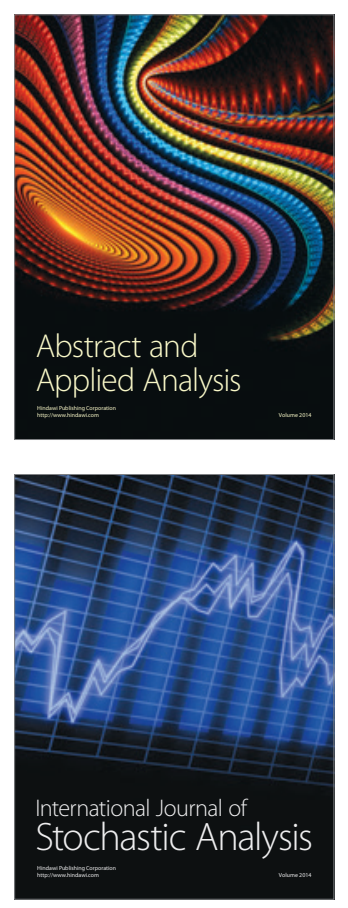

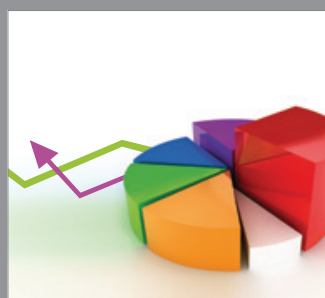

ournal of

Probability and Statistics

Promensencen
